\title{
Utilising Sodium-Mediated Ferration for Regioselective Functionalisation of Fluoroarenes via C-H and C-F Bond Activations
}

\author{
Lewis C. H. Maddock, ${ }^{[a]}$ Tracy Nixon, ${ }^{[a]}$ Alan R. Kennedy, ${ }^{[a]}$ Michael R. Probert, ${ }^{[b]}$ William Clegg, ${ }^{[b]}$ and \\ Eva Hevia*[a]
}

\begin{abstract}
Pairing iron bis(amide) $\mathrm{Fe}(\mathrm{HMDS})_{2}$ with $\mathrm{Na}(\mathrm{HMDS})$ to form new sodium ferrate base [(dioxane $\left.)_{0.5} \cdot \mathrm{NaFe}(\mathrm{HMDS})_{3}\right]$ (1) enables regioselective mono and di-ferration (via direct $\mathrm{Fe}-\mathrm{H}$ exchange) of a wide range of fluoroaromatic substrates under mild reaction conditions. Trapping of several ferrated intermediates has provided key insight into how synchronised $\mathrm{Na} / \mathrm{Fe}$ cooperation operates in these transformations. Furthermore using excess 1 at $80^{\circ} \mathrm{C}$ switches on a remarkable cascade process inducing the collective 2-fold $\mathrm{C}-\mathrm{H} / 3$-fold $\mathrm{C}-\mathrm{F}$ bond activations, where each $\mathrm{C}-\mathrm{H}$ bond is transformed to a $\mathrm{C}-\mathrm{Fe}$ bond whereas each $\mathrm{C}-\mathrm{F}$ bond is transformed into a $\mathrm{C}-\mathrm{N}$ bond.
\end{abstract}

Attracted by low cost, high natural abundance and low toxicity of the metal, organoiron chemistry continues to expand as increasing focus is directed towards sustainable practices. Though iron complexes have demonstrated proficiency in a myriad of cornerstone organic bond forming transformations under both stoichiometric and catalytic regimes, an area that remains surprisingly scant is deprotonative metallation. ${ }^{[1]}$ Metalhydrogen exchange (metallation) is one of the World's most practiced reactions. ${ }^{[2]}$ Alkyllithium or lithium amide reagents dominated metallation for decades, but recently new bimetallic systems have gained prominence as more efficient, more chemoselective alternatives. ${ }^{[3]}$ Only limited advances have been made in direct ferration of aromatic molecules. For example, Mongin used an in situ 1:3, $\mathrm{FeBr}_{2}$ :LiTMP stoichiometry for ferration of substituted pyridines, though $\mathrm{Fe}(\mathrm{II}) / \mathrm{Fe}(\mathrm{III})$ or $\mathrm{Fe}(\mathrm{II}) / \mathrm{Fe}(\mathrm{IV})$ couples were proposed to interfere, leading to homocoupling side reactions. ${ }^{[4]}$ Knochel similarly prepared trimetallic $(\mathrm{TMP})_{2} \mathrm{Fe} \cdot 2 \mathrm{MgCl}_{2} \cdot 4 \mathrm{LiCl}$ in situ to ferrate a range of functionalised arenes to form diaryl $\mathrm{Fe}(\mathrm{II})$ compounds. ${ }^{[5]}$ Significantly, the identities of the iron intermediates involved in this challenging chemistry were not identified. A case where structural elucidation proved possible was Mulvey's regioselective 2-fold ferration of benzene at the 1,4-positions by the sodium ferrate [(TMEDA) $\left.\mathrm{NaFe}(\mathrm{TMP})_{2}\left(\mathrm{CH}_{2} \mathrm{SiMe}_{3}\right)\right]$, which affords an iron-host inverse crown complex. ${ }^{[6]}$

While iron bis-amide, $\mathrm{Fe}(\mathrm{HMDS})_{2}$ [HMDS $=\mathrm{N}\left(\mathrm{SiMe}_{3}\right)_{2}$ ], has been reported as a useful precursor to access low-coordinate $\mathrm{Fe}$ complexes, ${ }^{[7]}$ the low polarity of its $\mathrm{Fe}-\mathrm{N}$ bonds makes it, a priori, unsuitable for $\mathrm{C}-\mathrm{H}$ metallation applications. Breaking new ground in this field, by pairing this seemingly toothless base with

[a] Dr. L.C. H. Maddock, Dr. T. Nixon, Dr. A. R. Kennedy, Prof. E. Hevia WestCHEM, Department of Pure and Applied Chemistry University of Strathclyde

295 Cathedral Street, Glasgow, G1 1XL (UK)

E-mail :eva.hevia@strath.ac.uk

[b] Dr. M. R. Probert, Prof. W. Clegg

Chemistry, School of Natural and Environmental Sciences,

Newcastle University, Newcastle upon Tyne, NE1 7RU, UK

Supporting information for this article is given via a link at the end of the document its sodium congener, we disclose (i) a new methodology for regioselective functionalisation of fluoroarenes which occurs via direct ferration of these aromatic scaffolds, and (ii) an unprecedented ate-mediated $\mathrm{C}-\mathrm{F}$ bond activation process for $\mathrm{C}$ $\mathrm{N}$ bond conversion.

While naturally occurring fluoroarenes are rare, synthetic forms are extremely valuable building blocks. Thus developing methods allowing the incorporation of these units into more complex molecular scaffolds is highly important. ${ }^{[8]}$ Interestingly, deprotonation of these molecules via organolithium bases still remains a challenge, primarily due to the exceptionally fragile stability of generated intermediates, which even at cryogenic conditions can eliminate LiF, to form benzyne intermediates as well as engaging in complex cascade processes involving autometallation. ${ }^{[9]}$ Also, even when low polarity metallators are used, fluoride elimination cannot be halted as shown recently by our group on alumination of fluoroarenes using trans-metaltrapping $\mathrm{Li} / \mathrm{Al}$ combinations, where metallated products decompose at room temperature. ${ }^{[10]}$

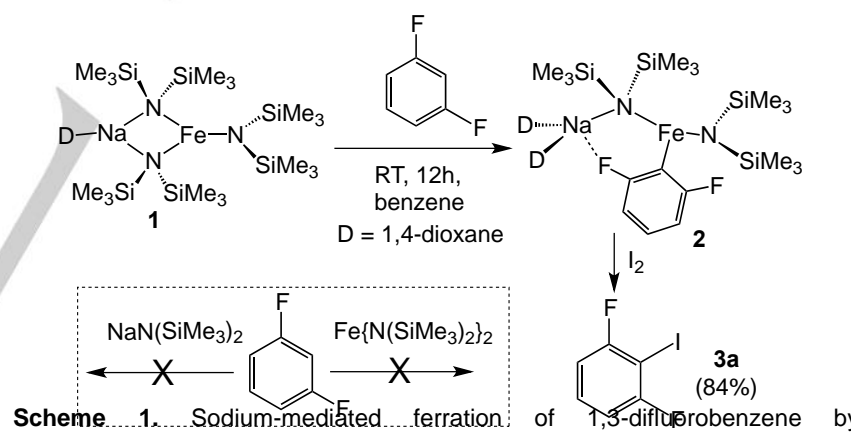
[(dioxane $\left.)_{0.5} \mathrm{NaFe}(\mathrm{HMDS})_{3}\right](\mathbf{1})$

We started the study by preparing the sodium tris(amido)ferrate [(dioxane $)_{0.5} \cdot \mathrm{NaFe}(\mathrm{HMDS})_{3}$ ] (1) in an isolated crystalline yield of $64 \%$ by co-complexing $\mathrm{Na}(\mathrm{HMDS}), \mathrm{Fe}(\mathrm{HMDS})_{2}$ and dioxane from a 1:1:1 mixture in benzene. ${ }^{[11]} \mathrm{X}$-ray crystallography confirmed the bimetallic constitution of $\mathbf{1}$, made up by two $\left\{\mathrm{NaFe}(\mathrm{HMDS})_{3}\right\}$ fragments connected via a Na-dioxane-Na bridge (see SI for details). Reacting 1,3-difluorobenzene with equimolar 1 at room temperature effected a colour change from a green to brown solution. Cooling this solution afforded heteroleptic [(dioxane) $\mathrm{NaFe}(\mathrm{HMDS})_{2}\left(1,3-\mathrm{F}_{2}-\mathrm{C}_{6} \mathrm{H}_{3}\right)$ ] (2) in $67 \%$ yield. In 2 the aromatic substrate is metallated at its $\mathrm{C} 2$ position between the $\mathrm{F}$ substituents. Electrophilic interception of $\mathbf{2}$ with iodine affords 1,3-difluoro-2-iodobenzene $3 a$ in $84 \%$ yield (Scheme 1).

In $2 \mathrm{Fe}$ forms a $\sigma$ bond at the 2-position of the aromatic ligand $[\mathrm{Fe}-\mathrm{C} 14,2.1157(18) \AA]$; whereas $\mathrm{Na}$ interacts with one $\mathrm{F}$ [Na-F1, 2.3437(13) A] (Fig 1).[12] Both metals are also connected by an 
HMDS bridge. Fe completes its coordination sphere by bonding to a terminal HMDS, while $\mathrm{Na}$ is solvated by two dioxane molecules.

Emphasising the cooperativity between sodium and iron inherent in the reactivity of mixed-metal base 1, neither homometallic $\mathrm{Na}(\mathrm{HMDS})$ or $\mathrm{Fe}(\mathrm{HMDS})_{2}$ on their own can deprotonate 1,3difluorobenzene (Scheme 1). Thus 2 can be regarded as a product of sodium-mediated ferration, with $\mathrm{Fe}$ actioning the deprotonation, but sodium being essential for this reaction to occur. Interestingly, it should also be noted that ferrated intermediate 2 is remarkably robust and does not decompose even when refluxed in $\mathrm{C}_{6} \mathrm{D}_{6}$ at $80^{\circ} \mathrm{C}$ for $24 \mathrm{~h}$.

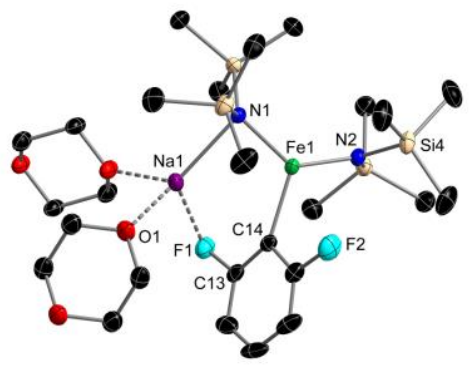

Figure 1 Monomeric, dinuclear structure of ferrate 2 with $\mathrm{H}$ atoms omitted for clarity and displacement ellipsoids displayed at $50 \%$ probability level.

Encouraged by this initial reactivity, we extended this approach to a range of fluoroaromatic substrates. Reactions were carried out in benzene, allowing mixtures to stir overnight prior to $\mathrm{I}_{2}$ quenching (Table 1). Each metallation occurred at a position adjacent to at least one fluorine atom, affording iodofluoroarenes $\mathbf{3 b - 1}$ in good to excellent yields (40-91\%). While Holland has reported that $\mathrm{Fe}(\mathrm{II})$ complexes can promote $\mathrm{C}-\mathrm{F}$ bond activation processes when treated with perfluorinated arenes, ${ }^{[13]}$ here, selective $\mathrm{Fe}-\mathrm{H}$ exchange reactions are observed. This $\mathrm{C}-\mathrm{H}$ chemoselectivity also contrasts with previous studies using $\mathrm{Fe}(0)$ systems where $\mathrm{C}-\mathrm{F}$ bond activation is usually preferred. ${ }^{[14]}$ Consistent with the different degree of $C$ $\mathrm{H}$ bond activation in the fluoroarenes studied, ${ }^{[15]}$ those substrates with only one $\mathrm{F}$ atom required harsher reaction conditions $\left(50^{\circ} \mathrm{C}\right)(\mathbf{3 b}, \mathbf{3 e}, \mathbf{3 k}$ and $\mathbf{3} \mathbf{l}$ in Table $1,40-74 \%$ yields), whereas other $\mathrm{Fe}-\mathrm{H}$ exchanges occur at $\mathrm{RT}$ or $0^{\circ} \mathrm{C}(\mathbf{3 c}, 3 \mathrm{~d}$ and 3f-3i in Table 1, yields 58-91\%). Interestingly, even in substrates possessing other ortho-directing groups such as 4-fluoroanisole or 4-fluorobenzonitrile, ortho-metallation at the $\mathrm{F}$ substituent is preferred (3k and $\mathbf{3} \mathbf{I}$ in Table 1 ). These regioselectivities are opposite to those reported for lithiation of 4-fluoroanisole by ${ }^{n} \mathrm{BuLi}$ in THF at $-78^{\circ} \mathrm{C}^{[16]}$ or for 4-fluorobenzonitrile using related heterobimetallic $(\mathrm{TMP})_{2} \mathrm{Fe} \cdot 2 \mathrm{MgCl}_{2} \cdot 4 \mathrm{LiCl} .^{[5]}$ Contrasting with many lithiation reactions, where excess metallating reagent leads to complex mixtures of products, ${ }^{[17]}$ here stoichiometric control is seen as two equivalents of 1 regioselectively di-ferrate 1,3,5-trifluorobenzene, 1,4-dibromo-2,5-difluorobenzene and 1,2,5,6-tetrafluorobenzene, furnishing $\mathbf{3 h}, \mathbf{3 i}$ and $\mathbf{3} \mathbf{j}$ in 68,85 and $88 \%$ yields respectively.

Interestingly, $\mathbf{1}$ failed to react with related chloroarenes, which also contain relatively activated $H$ 's, such as 1,3 dichlorobenzene, even under harsh conditions $\left(50^{\circ} \mathrm{C}, 24 \mathrm{~h}\right)$. This lack of reactivity contrasts with Schlosser's work using ${ }^{\mathrm{s} B u L i}$ at $-100^{\circ} \mathrm{C}$, where competition studies between this substrate and fluorobenzene show a clear preference for the former. ${ }^{[18]}$

Table 1 Ferration of fluoroarenes using sodium ferrate $1^{\text {[a] }}$

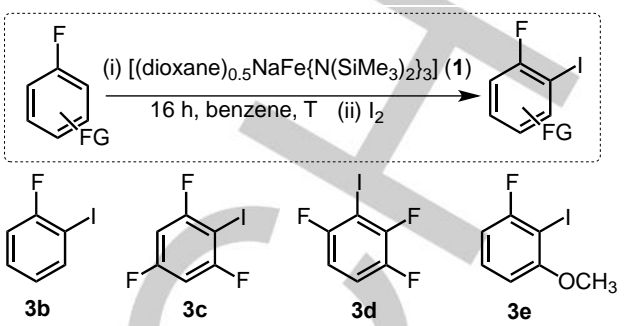

$\left(50^{\circ} \mathrm{C}, 74 \%\right)^{[\mathrm{b}]} \quad\left(0^{\circ} \mathrm{C}, 58 \%\right)^{[\mathrm{c}]} \quad(\mathrm{RT}, 80 \%) \quad\left(50^{\circ} \mathrm{C}, 78^{\circ}\right)$

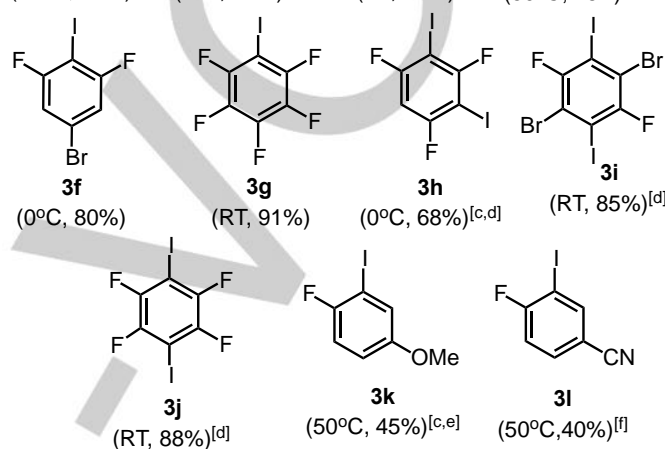

[a] Reactions carried out with in situ prepared 1 in benzene. Unless otherwise stated, stoichiometric amounts of base and substrate and a five molar excess of iodine were used (see SI for details). Yields are of isolated products, see SI for details. [b] Fluorobenzene was used as solvent in the reaction. [c] Yield determined by ${ }^{1} \mathrm{H}$ NMR using ferrocene as internal standard. [d] Carried out using 2 equivalents of 1 . $^{[\mathrm{e}]} 5$ days, using 2 equivalents of 1 . $^{[\mathrm{fl}} 2$ days.

To shed more light on these intriguing findings, we next investigated key metallated intermediates $\mathbf{4 a}$ and $\mathbf{4 b}$ (Fig. 2), resulting from diferration of 1,3,5-trifluorobenzene and 1,2,4,5tetrafluorobenzene prior to electrophilic interception.



Figure 2 Molecular structures of complexes $\mathbf{4 a}$ (left) and $\mathbf{4 b}$ (right). Hydrogen atoms (except $\mathrm{H} 4$ in $\mathbf{4 a}$ ), minor disorder at one $\mathrm{SiMe}_{3}$ in $\mathbf{4 b}$ and a molecule of co-crystallised benzene solvent in $\mathbf{4 a}$ are omitted for clarity. Displacement ellipsoids displayed at $30 \%$ probability level. ${ }^{[19]}$

Both structures bear a strong resemblance to that of $\mathbf{2}$, with the $\mathrm{Fe}$ centres occupying positions previously filled by $\mathrm{H}$ atoms (mean Fe-C distance, 2.110 and $2.134 \AA$ for $\mathbf{4 a}$ and $\mathbf{4 b}$ respectively), whereas each $\mathrm{Na}$ atom binds datively with one $\mathrm{F}$ at the ortho positions (mean Na-F distance, 2.335 and $2.297 \AA$ for $\mathbf{4} \mathbf{a}$ and $\mathbf{4} \mathbf{b}$ respectively). The distinct bonding preferences of $\mathrm{Fe}$ and $\mathrm{Na}$ in these structures, tying up $\mathrm{C}$ and $\mathrm{F}$ lone pairs respectively, must contribute to the unusual room temperature stability of these potentially hypersensitive fluoroaryl dianions. Steric stabilization of the Fe-C bonds via the bulky HMDS 
groups is presumably another factor. As far as we can ascertain, 4a represents the first structurally defined example of dimetallation of 1,3,5-trifluorobenzene whereas for 1,2,4,5tetrafluorobenzene only two examples have been reported, though neither were made via direct metal- $\mathrm{H}$ reactions. ${ }^{[20]}$

Previous studies of heterobimetallic amide bases such as alkalimetal zincates have shown that metallation regioselectivities can be influenced by coordination of the substrate to the alkali-metal prior to the metallation step. ${ }^{[21]}$ A similar scenario could be envisaged here, where initially the fluoroarene could coordinate to $\mathrm{Na}$ in $\mathbf{1}$ via a dative $\mathrm{Na}-\mathrm{F}$ bond similar to those seen in $\mathbf{2 , 4 a}$ and $\mathbf{4 b}$, bringing its adjacent $\mathrm{H}$ into close proximity to the anionically-activated ferrate $\left\{\mathrm{Fe}(\mathrm{HMDS})_{3}\right\}$ moiety, priming the intramolecular $\mathrm{Fe}-\mathrm{H}$ exchange. $\mathrm{Na} \cdots \mathrm{F}$ interactions could therefore hold the key to the ortho-F selectivity/reactivity witnessed for all substrates studied (vide supra), whereas lack of such interactions could be a factor in the inertness of $\mathbf{1}$ with chloroarenes.

Exploring the ferrating limits of $\mathbf{1}$, we next pondered if the three $\mathrm{H}$ atoms in 1,3,5-trifluorobenzene are exchangeable by $\mathrm{Fe}$ centres. Thus reacting the fluoroarene with excess 1 at $80^{\circ} \mathrm{C}$ (Scheme 2) gave a solution colour change from green to dark yellow and the formation of a precipitate. Cooling this solution gave yellow crystals of unimetallic iron complex [1,3$\left.\{\mathrm{Fe}(\mathrm{HMDS})\}_{2}-2,4,6-(\mathrm{HMDS})_{2}-\mathrm{C}_{6} \mathrm{H}\right](5 \mathrm{a})$ in $87 \%$ yield, where the fluoroarene has undergone an extraordinary 2 -fold $\mathrm{C}-\mathrm{H} / 3$-fold $\mathrm{C}$ $\mathrm{F}$ activation, five bond-breaking process (Scheme 2). ${ }^{[22]}$ This approach could also be extended to 1,2,3,5-tetrafluorobenzene and 1-bromo-2,4,6-trifluorobenzene, furnishing bis(iron) compounds $\mathbf{5 b}$ and $\mathbf{5 c}$ (Scheme 2 ).

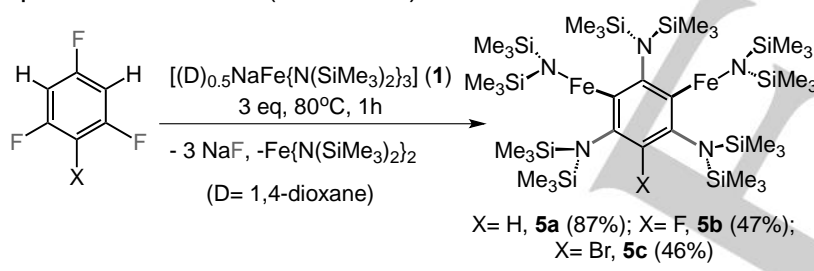

Scheme 2. Two-fold $\mathrm{C}-\mathrm{H} /$ three-fold $\mathrm{C}-\mathrm{F}$ activation processes of $1,3,5$ trifluorobenzenes.

The molecular structures of $\mathbf{5 a - c}$ were established by $\mathrm{X}$-ray crystallographic studies (see SI for full details and Fig. 3 for $5 a$ ). Since $\mathbf{5 a - c}$ are isostructural, only $\mathbf{5 a}$ is discussed. Containing a 1,3-diferrated-pentasubstituted aryl ring, where each $\mathrm{F}$ atom of 1,3,5-trifluorobenzene has been replaced by HMDS, 5a displays two low-coordinate $\mathrm{Fe}$ centres, each bonded to one $\mathrm{C}$ of the aromatic ring (mean $\mathrm{Fe}-\mathrm{C}$ distance, $2.024 \AA$ ) and a terminal HMDS, adopting an unusual near-linear coordination (with C-Fe$\mathrm{N}$ bond angles of 159.43(1) and 161.06(12) ${ }^{\circ}$ for Fe1 and Fe2 respectively). Each $\mathrm{Fe}$ also forms a long-range contact with a neighboring HMDS attached to the aryl ring (mean Fe $\cdots \mathrm{N}(\mathrm{HMDS})_{\text {ring }}, 2.389 \AA$ ). While these contacts are notably more elongated than those observed for HMDS groups directly attached to $\mathrm{Fe}$ (mean Fe-N distance, $1.919 \AA$ ) , they are significant enough to induce the distortion from linearity observed in the Fe geometries. It should be noted that twocoordinate iron (II) complexes are scarce in coordination chemistry, although they have already demonstrated unique magnetic properties. ${ }^{[23]}$ As far as we can ascertain $5 \mathbf{a}-\mathbf{5 c}$ are the first dinuclear systems containing two pseudo-linear Fe centres to be structurally defined.

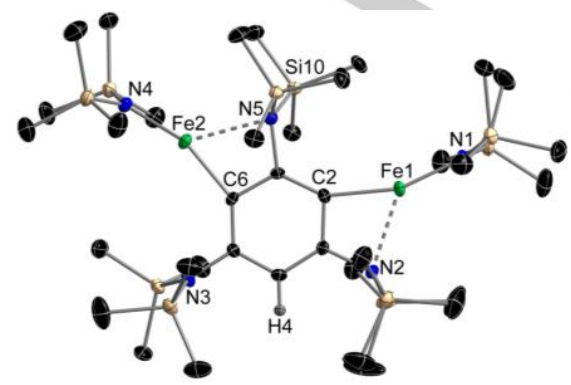

Figure 3 Molecular structure of 5a. Hydrogen atoms (except H4) are omitted for clarity. Displacement ellipsoids displayed at $50 \%$ probability level.

While C-F bond activation of fluoroarenes by transition metals has been much investigated, to date, iron has shown limited promise in this area. ${ }^{[8,12,14]}$ It should also be noted that methods for C-F/C-N conversion are scarce, confined mainly to nucleophilic aromatic substitution of activated fluoroaromatics. ${ }^{[2]}$ Here, the synergic power of this $\mathrm{Na} / \mathrm{Fe}$ bimetallic partnership allows conversion of three $\mathrm{C}-\mathrm{F}$ fluoroarene bonds into three new $\mathrm{C}-\mathrm{N}$ bonds as well as two direct $\mathrm{Fe}-\mathrm{H}$ exchanges (Scheme 2). Filtrate analysis from the reaction of 1,3,5trifluorobenzene and 3 equivalents of 2 showed the presence of $\mathrm{Fe}(\mathrm{HMDS})_{2} \cdot($ dioxane) complex and $\mathrm{HMDS}(\mathrm{H})$, while formation of precipitated $\mathrm{NaF}$ was confirmed by ${ }^{19} \mathrm{~F} \mathrm{NMR}$ in $\mathrm{D}_{2} \mathrm{O}$. Furthermore, it was found that $\mathbf{5 a}$ can also be prepared in excellent yield (90-95\%) by treating diferrated product $\mathbf{4 a}$ with one equivalent of sodium ferrate 1 or just one equivalent of $\mathrm{Na}(\mathrm{HMDS})$ at $80^{\circ} \mathrm{C}$. Even more revealing, heating $4 \mathrm{a}$ on its own in benzene for over an hour at $80^{\circ} \mathrm{C}$ also furnished $\mathbf{5 a}$ albeit in a significantly lower yield (58\%).

While this type of reactivity is unprecedented in transition-metal chemistry, Schlosser reported the conversion of 1,3,5trifluorobenzene into 1,3,5-tris(tert-butyl)benzene using excess ${ }^{t} \mathrm{BuLi}$ via dilithiated aryl intermediates. ${ }^{[9]}$ Reflecting on that and our experimental findings, we propose that C-F bond activation products $\mathbf{5}$ form as a consequence of a cascade process, which takes place at $80^{\circ} \mathrm{C}$. In a first step, differration of the fluoroaryl (e.g. 1,3,5-trifluorobenzene in Scheme 3) by 2 equivalents of 1 will occur to form sodium ferrate $\mathbf{4 a}$. While this compound is stable at room temperature (vide supra), at $80^{\circ} \mathrm{C}$ it reacts to give $5 a$, via an unstoppable domino of alternating $\mathrm{NaF}$ and $\mathrm{Fe}(\mathrm{HMDS})_{2}$ eliminations and subsequent $\mathrm{Fe}(\mathrm{HMDS})_{2}$ additions to benzyne intermediates. The existing $\mathrm{Na}$...F contacts in $\mathbf{4 a}$ may contribute to facilitate the activation of the relevant $\mathrm{C}-\mathrm{F}$ bonds under these reaction conditions. ${ }^{[25]}$ Each diferrated intermediate should be less stable than its precursor (III>II>I $>\mathbf{4 a}$ in Scheme 3), as this fast sequence of reactions does not stop until the three $\mathrm{F}$ atoms are replaced by HMDS groups, affording 5a, where now the low-coordinate $\mathrm{Fe}$ centres are protected by bulky HMDS groups on the aryl ring. ${ }^{[26]}$ It should be noted that to facilitate the last $\mathrm{NaF} / \mathrm{Fe}(\mathrm{HMDS})_{2}$ elimination step $(\mathrm{III} \rightarrow \mathbf{5 a}$, Scheme 3), requires co-complexation of II with one $\mathrm{Na}$ (HMDS) equivalent. The sodium amide can be provided by a third equivalent of $\mathbf{1}$ (which explains the detection of 
$\mathrm{Fe}(\mathrm{HMDS})_{2} \cdot($ dioxane) complex with three equivalents of $\mathbf{1}$ ), by some unreacted $\mathbf{4 a}$ or by adding an extra equivalent of $\mathrm{Na}(\mathrm{HMDS})$ to $4 \mathrm{a}$. Interestingly, contrasting with these results, when diferrated intermediate $\mathbf{4 b}$, whose Fe centres reside at the 1,4-positions of the fluoroaryl anion, is reacted with an excess of 1 and heated at $80^{\circ} \mathrm{C}$, no reaction was observed, hinting that the proximity of the Fe centres within the ring must also play a role in facilitating this activation process.

$$
\text { differation }
$$

Scheme 3. Proposed cascade mechanism for formation of $5 a(D=$ dioxane, $\mathrm{R}=\mathrm{SiMe}_{3}$ ).

To conclude, by exploiting synergistic effects in sodium ferrate chemistry, we have unveiled a new regioselective method for the direct ferration of a wide range of fluoroarenes under mild reaction conditions as well as a novel type of C-F bond activation process. Isolation of metallated intermediates have shed light on how $\mathrm{Na}$ ions and Fe centres can cooperate in a synchronised manner to promote chemical transformations which neither sodium or iron amides are capable of facilitating on their own.

\section{Acknowledgements}

We are grateful to the ERC (Stg-2011 MixMetApps to EH) for funding and to Diamond Light Source for access to single-crystal synchrotron diffraction facilities on beamline 119 (award MT11145). We also thank Professor Robert E. Mulvey and Dr. Charles T. O'Hara for their insightful comments.

Keywords: metallation, iron, mixed-metal chemistry; C-F bond activation, alkali-metals

[1] For an authoritative review on Fe-mediated $\mathrm{C}-\mathrm{H}$ bond activation see: $\mathrm{R}$. Shang, L. llies, E. Nakamura, Chem. Rev. 2017, 117, 9086.

[2] J. Clayden, Organolithiums: Selectivity for Synthesis, Pergamon, Elsevier Science Ltd., Oxford, 2002.

[3] F. Mongin, A. Harrison-Marchand, Chem. Rev. 2013, 113, 7563.

[4] E. Nagaradja, F. Chevallier, T. Roisnel, V. Jouikov, F. Mongin, Tetrahedron, 2012, 68, 3063

[5] S. H. Wunderlich, P. Knochel, Angew. Chem. 2009, 121, 9897; Angew. Chem. Int. Ed. 2009, 48, 9717
[6] P. Albores, L. M. Carella, W. Clegg, P. Garcia-Alvarez, A. R. Kennedy J. Klett, R. E. Mulvey, E. Rentschler, L. Russo, Angew. Chem. 2009, 121, 3231; Angew. Chem. Int. Ed. 2009, 48, 3317.

[7] C. G. Werncke, P. C. Bunting, C. Duhayon, J. R. Long, S. Bontemps, S Sabo-Etienne, Angew. Chem. 2014, 127, 247; Angew. Chem. Int. Ed. 2014, 54, 245.

[8] T. Ahrens, J. Kohlmann, M. Ahrens, T. Braun, Chem. Rev. 2015, 115, 931.

[9] M. Schlosser, L. Guio, F. Leroux, J. Am. Chem. Soc. 2001, 123, 3822.

[10] R. McLellan, M. Uzelac, A. R. Kennedy, E. Hevia, R. E. Mulvey, Angew. Chem. 2017, 129, 9694; Angew. Chem. Int. Ed. 2017, 56, 9566.

[11] a) L. C. H Maddock, T. Cadenbach, A. R. Kennedy, I. Borilovic, G. Aromi, E. Hevia, Inorg. Chem. 2015, 54, 9201. b) L. C. H Maddock, I. Borilovic, J. Mclntyre, A. R. Kennedy, G. Aromi, E. Hevia, Dalton Trans. 2017, 46, 6683.

[12] For a review on the coordination chemistry of the CF unit in fluorocarbons see: H. Plenio, Chem. Rev. 1997, 97, 3363.

[13] J. Vela, J. M. Smith, Y. Yu, N. A. Ketterer, C. J. Flaschenriem, R. J. Lachicotte, P. L. Holland, J. Am. Chem. Soc. 2005, 127, 7857.

[14] For a reviews on competition between $\mathrm{C}-\mathrm{H} / \mathrm{C}-\mathrm{F}$ bond activations see: $\mathrm{O}$ Eisenstein, J. Milani, R. N. Perutz, Chem. Rev. 2017, 117, 8710.

[15] Hyla-Kryspin, S. Grimme, H. H. Büker, N. M. M. Nibbering, F. Cotter, M. Schlosser, Chem. Eur. J. 2005, 1251.

[16] M. Schlosser, Angew. Chem. 1998, 110, 1538; Angew. Chem. Int. Ed. 1998, 110, 1496.

[17] A. Orthaber, C. Seidel, F.Belaj, Jörg H. Albering, R. Pietschnig, U. Ruschewitz, Inorg. Chem. 2010, 49, 9350

[18] M. Schlosser, E. Marzi, F. Cottet, H. H. Büker, N. M. M. Nibbering Chem. Eur. J. 2001, 3511.

[19] $\mathbf{4 a}$ and $\mathbf{4 b}$ form complex 2D-network structures with propagation occurring via their dioxane molecules which connect to neighbouring $\left[\left(\mathrm{Na}_{2} \mathrm{Fe}_{2}(\mathrm{HMDS})_{4}\right.\right.$ (diarenide)] units, see SI.

[20] a) T. Schaub, P. Fischer, T. Meins, U. Radius, Eur. J. Inorg. Chem 2011, 3122. b) R. Chukwu, A. D. Hunter, B. D. Santarsiero, J. L. Atwood, J. Chassaignac, Organometallics, 1992, 11, 589.

[21] D. R. Armstrong, V. L. Blair, W. Clegg, S. H. Dale, J. Garcia-Alvarez, G W. Honeyman, E. Hevia, R. E. Mulvey, L. Russo, J. Am. Chem. Soc. 2010, 132, 9480.

[22] Hydrolysis of $\mathbf{5 a}$ afforded tri-substituted benzene [1,3,5-(HMDS) $\left.{ }_{3}-\mathrm{C}_{6} \mathrm{H}_{3}\right]$ (6) in a $71 \%$ yield which was fully characterized by ${ }^{1} \mathrm{H},{ }^{13} \mathrm{C} N M R$ and elemental analysis. Its solid structure was established by X-ray crystallographic studies (see SI for details).

[23] P. P. Power, Chem. Rev. 2012, 112, 3482 and references therein.

[24] a) X. Xu, J. Jia, H.Sun, Y. Liu, W. Xu, Y. Shi, D. Zhang, X. Li, Dalton Trans., 2013, 42, 3417. b) T. Zheng, J. Li, S. Zhang, B. Xue, H. Sun, X. Li, O. Fuhr, D. Fenske, Organometallics, 2016, 35, 3538.

[25] Li...F interactions have been proposed to play a major role in the $\mathrm{C}-\mathrm{F}$ bond activation of $\mathrm{CF}_{3} \mathrm{H}$ by Li enolates, see: T. lida, R. Hashimoto, $\mathrm{K}$ Aikawa, S. Ito, K. Mikami, Angew. Chem. 2012, 124, 9673; Angew. Chem. Int. Ed. 2012, 51, 9535.

[26] Attempts to trap any of the proposed intermediates by hydrolysis resulted in the isolation of variable amounts of $\left[1,3,5-(\mathrm{HMDS})_{3}-\mathrm{C}_{6} \mathrm{H}_{3}\right](6)$ (see SI) and 1,3,5-trifluorobenzene. 
Entry for the Table of Contents (Please choose one layout)

Layout 2:

\section{COMMUNICATION}



High Five! Exploiting bimetallic cooperation, sodium tris(amido) ferrate 1 induces the collective cleavage of five (three $\mathrm{C}-\mathrm{F}$ and two $\mathrm{C}-\mathrm{H}$ ) bonds of trifluoroarene substrates via a cascade activation process.
L. C. H. Maddock, T. Nixon, A. R. Kennedy, M. R. Probert, W. Clegg and E. Hevia*

Page No. - Page No.

Utilising Sodium-Mediated Ferration for Regioselective Functionalisation of Fluoroarenes via $\mathbf{C}-\mathrm{H}$ and $\mathrm{C}-\mathrm{F}$ Bond Activations 